\title{
IoT System for Anytime/Anywhere Monitoring and Control of Vehicles' Parameters
}

\author{
Jose A. Afonso and \\ Ruben A. Sousa \\ CMEMS-UMinho Center \\ University of Minho \\ Campus of Azurem \\ 4800-058 Guimaraes, Portugal
}

\author{
Joao C. Ferreira \\ Information Sciences, Technologies \\ and Architecture Research Center \\ Instituto Universitário de Lisboa \\ (ISCTE-IUL) \\ 1649-026 Lisboa, Portugal
}

\author{
Vitor Monteiro, Delfim Pedrosa \\ and Joao L. Afonso \\ Centro ALGORITMI \\ University of Minho \\ Campus of Azurem \\ 4800-058 Guimaraes, Portugal
}

\begin{abstract}
This paper presents an IoT (Internet of Things) system designed to allow the monitoring and control of parameters of the users' vehicles, anytime and anywhere in the world, through the Internet. The system prototype was developed and tested using an electric vehicle (EV) and the respective sensor systems. The main components of the proposed IoT system are: a Bluetooth Low Energy (BLE) intra-vehicular wireless sensor network (IVWSN); a mobile device that acts both as the vehicle's gateway, connecting the IVWSN to the Internet, and as the vehicle's human machine interface (HMI); an online server/database, based on Firebase; a client, which can be either a mobile device or a personal computer; and a residential wireless sensor network (WSN). The use of a wireless network to collect sensor data inside of the vehicle introduces some advantages when compared with conventional wired networks, whereas the inclusion of a residential WSNs in the proposed IoT architecture allows the provision of additional features, such as automatic control of the $\mathrm{EV}$ battery charging process. Experimental results are provided to assess the performance of the developed IVWSN and HMI.
\end{abstract}

Keywords-Internet of Things, Bluetooth Low Energy; Electric Vehicles, Monitoring and Control, Wireless Sensor Networks

\section{INTRODUCTION}

Mobile data systems are getting cheaper and more widespread each year. People are staying online longer than before, which opens up tremendous possibilities for projects related to the Internet of Things (IoT) [1]. Around 47\% of the world's population is already using the Internet [2] and by 2020 it is foreseen that the number of devices connected to the Internet will be over 50 billion [3]. If the world's population increases to 8 billion in that same year, this means we will have more than six devices connected to the Internet per human being.

Users now may have access to a multitude of data from multiple sources that were unavailable before, such as physiological or biometric data gathered with a wristband device or a watch. IoT opens up possibilities that were until recently only in the realm of sci-fi movies. Today, we are starting to build smart homes, cities and vehicles that manage and control themselves without needing user input.
The main components of an IoT system usually are [4]: 1) Embedded sensor/actuator devices (the "things" in IoT); 2) A gateway (also called hub), which is required when the embedded devices do not have native IP connectivity; 3) An Internet service/database running on an online Web server; 4) One or more clients (either app or browser-based), for the users to interact with the service via mobile phones, tablets or personal computers (PCs). The gateway has two main functions: 1) Translate the packets between the embedded device protocols (e.g., ZigBee, Z-Wave and/or Bluetooth) and the TCP/IP protocol; 2) Provide access to the Internet. In many cases the gateway can be co-located with the local Internet router, sharing the utilization with conventional (non IoT) traffic such as Web browsing.

The gateway on the IoT architecture can be a fixed, dedicated device, such as the Samsung SmartThings Hub. In alternative, it can be a mobile, shared device, such as a smartphone. In this case, data can be collected either from the mobile device's internal sensors (e.g., microphone, camera, GPS (Global Positioning System), accelerometer, magnetometer, gyroscope, proximity sensor or light sensor) or through external sensor devices, which can be connected to the mobile device using wired (e.g., USB) or wireless technologies (e.g., Bluetooth, ANT, etc.). These external devices may be placed on the user's body (e.g., heart rate monitor, posture sensors [5]) or in the environment (e.g., air temperature). Mobile phone sensing systems are an emerging area of research and development potentiated by the widespread proliferation of mobile devices [6]. A third IoT architecture alternative consists of the direct connection of the embedded devices to the Internet, eliminating the necessity of a gateway. Examples are devices that possess connectivity to mobile data cellular networks or an IEEE 802.11/Wi-Fi interface, such as the Belkin WeMo devices.

Sensor/actuator devices in modern vehicles are normally connected using a cabled network technology called CAN (Controller Area Network) [7]. The increasing number of sensors on vehicles also increases the number of cables required and, consequently, the weight and maintenance costs. The cables also impose constraints on the placement of sensors. The use of wireless technologies has the potential to solve these problems [8] and to increase the flexibility and mobility 
of the system, allowing also the installation of sensors in the vehicle on demand, when and where desired, as well as the integration of wearable sensors [9] to monitor the drivers' biomedical signals and even to provide alerts when the users are not able to drive.

This paper presents an IoT system applied to transportation designed to allow the monitoring and control of parameters of the users' vehicles anywhere in the world through the Internet. The main components of the system are a Bluetooth Low Energy (BLE) intra-vehicular wireless sensor network (IVWSN), a vehicle's gateway and human machine interface (HMI), an online server/database, a mobile client and a residential wireless sensor network (WSN) [10].

Electric vehicles (EVs) are the future of transportation systems. The sales of these vehicles keep increasing throughout the years and, according to [11], by the year 2022 they will cost the same as the traditional non-ecofriendly vehicles. Battery prices are also decreasing [12], which also helps in increasing the sales. The system proposed in this work was developed using the CEPIUM (Carro Elétrico Plug-In da Universidade do Minho - in Portuguese language) electric vehicle [13], shown in Fig. 1. This car was developed by the Group of Energy and Power Electronics (GEPE) of University of Minho, Portugal, based on a Volkswagen Polo, where the internal combustion engine parts were replaced by an electric motor, batteries and required electronic circuits.

This paper is organized as follows. Section II presents related work regarding BLE networks and IoT systems. Section III presents the architecture of the IoT system proposed in this paper and a description of its main components. Section IV presents experimental results concerning the developed system. Finally, Section V presents the conclusions.

\section{RELATED WORK}

Bluetooth Low Energy is one of the most promising current wireless technologies to satisfy the requirements posed by IVWSNs [8], such as low energy consumption [14], low latency, low cost and high reliability. BLE is also considered as one of the most promising technologies enabling the IoT [15].

In [16], the authors conclude that BLE presents very high energy efficiency in terms of energy per bit when compared to IEEE 802.15.4/ZigBee. In [17], the authors present experimental results that indicate that BLE outperforms ZigBee in vehicles under the interference of Wi-Fi networks. In [18], the authors study the use of BLE notifications and conclude that BLE is able to support several sensor nodes generating high data rate traffic and that it outperforms IEEE 802.15.4based protocols in terms of energy consumption and number of supported nodes.

The integrated support on most smartphones is another advantage of BLE over alternative technologies such as IEEE 802.15.4/ZigBee. All these reasons motivated the choice of BLE for the development of the system described in this paper.

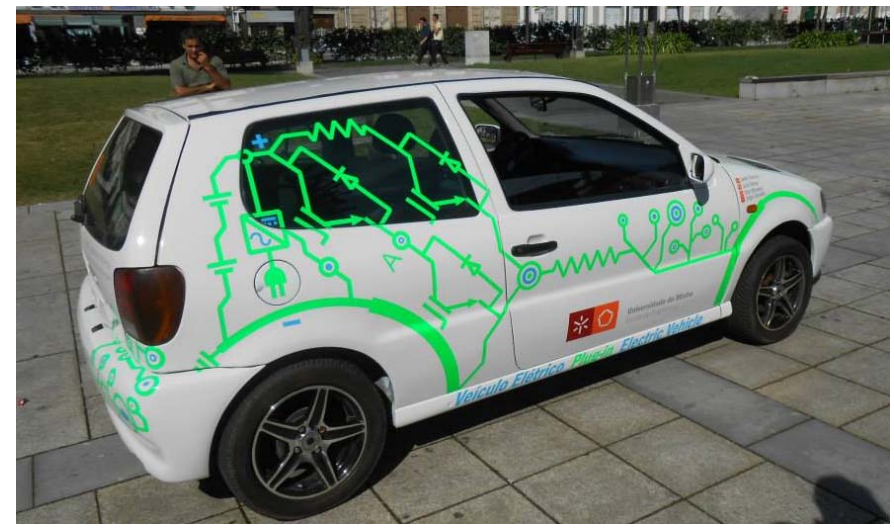

Fig. 1. Electric vehicle used in the development of the IoT system.

Android Auto is an extension of the mobile operating system Android for vehicles [19]. The main purpose of Android Auto is to replace the need of using a smartphone inside a car. Some compatible cars allow the user to display the smartphone screen in the dashboard's head unit. In case there is no display in the car, users can still use their smartphone with the app Android Auto. Support for reading data from the car sensors (velocity, fuel, etc.), which is still in development, may allow tighter integration in the future and increase the usefulness of this system.

In [20], the authors present an IoT middleware solution for a smart home system that was developed. The architecture consists of a set of sensors for appliances, the GSN (Global Sensor Networks) middleware [21] to retrieve and process the sensors' data, a backend as a service (BaaS), called Firebase [22], to store the data and a website to visualize the sensors' data. The GSN connects to the sensors and forwards the data to the Firebase database. Meanwhile, the users can see changes on the website in real-time through a connection to the Firebase server.

In [23], the authors present a vehicle diagnostic and tracking system where the on-board diagnostic (OBD) system is used to retrieve the vehicle's data. The architecture implemented shares some similarities to the one proposed in this paper; however, unlike our work, this system does not implement a wireless network connecting multiple sensors and actuators distributed inside the vehicle. An interface controller is used to connect the OBD system to a Bluetooth controller, which then connects to an Android-based mobile application that synchronizes the collected data with a Web server. The application is also able to configure parameters that include enabling/disabling GPS and set the refresh period of the data synchronization.

In [24], the architecture of a decentralized charging system for residential buildings was proposed. According to this proposal, a charging management system would be placed in each residential area and would act on real-time data provided from the Internet. The charging would depend on three factors: user convenience, variation of electricity prices and the driver's paying availability. The goal is to provide a better choice for users and also to help decreasing electricity consumption. 
In [25], a system for dynamic charging of EVs batteries was proposed for smart homes integration. The system enables or disables the charging process depending on the current load in the house. If the load is too high, i.e., more than the acceptable for the electrical installation, the system starts operating in vehicle-to-grid (V2G) mode, and the battery starts delivering power to the grid. Otherwise, if the load is low, the system operates in home-to-vehicle $(\mathrm{H} 2 \mathrm{~V})$ mode and the battery starts charging using power from the house and according to the loads current consumption.

\section{SYSTEM OVERVIEW}

The main components of the proposed IoT system for vehicles (Fig. 2) are an intra-vehicular wireless sensor network (one per vehicle), the vehicle's gateway and HMI, which connects to the Internet, an online server/database (which may serve several users/vehicles), a client mobile app and the user's home WSN, which is also connected to the Internet.

\section{A. Intra-Vehicular Wireless Sensor Network}

The IVWSN was implemented using BLE. As shown in Fig. 3, it is comprised of two types of BLE nodes: a central station (smartphone or tablet), which is located at the car dashboard, near the driver, and several peripheral stations spread around the vehicle.

Each peripheral station, in turn, is composed of a BLE module and a sensor module. The current prototype uses Texas Instruments (TI) CC2540EM BLE modules. Fig. 4 shows one of these modules at the top, with its antenna, which is connected to a SmartRF05EB evaluation board. Connectors P18 and P20 provide access to the pins of the CC2450 system-on-chip (SoC) of the CC2540EM module, allowing the connection of external sensors via ADC (Analog-to-Digital Converter), UART (Universal Asynchronous Receiver/Transmitter) or SPI (Serial Peripheral Interface). Although the SmartRF05EB board is much larger when compared to the CC2540EM module, it is only needed during the development phase. The embedded software in the BLE modules was developed in $\mathrm{C}$ using the IAR Embedded Workbench IDE (Integrated Development Environment).

The sensor modules can range from single sensors with ADC, UART or SPI interface (e.g., a temperature sensor), to complex systems composed of several sensors sharing a common interface. Currently, there are two of these sensor systems implemented in our EV: the traction system, placed inside of the hood, and the battery system, located below the rear seats (Fig. 3). The traction system provides data related to the parameters of the electric motor controller, the state of charge and the temperature of the motor. The battery system provides information related to the battery and the electric charger. These systems transmit their data to the respective BLE module via UART, except for the motor temperature, which uses the BLE module ADC. A polling protocol was implemented, where each BLE module acts as master and the respective sensor system acts as slave. The data frame sent by the traction system has a header (composed of the following fields: system address, frame type and frame length) and a payload (which contains the samples from its 6 sensors). The

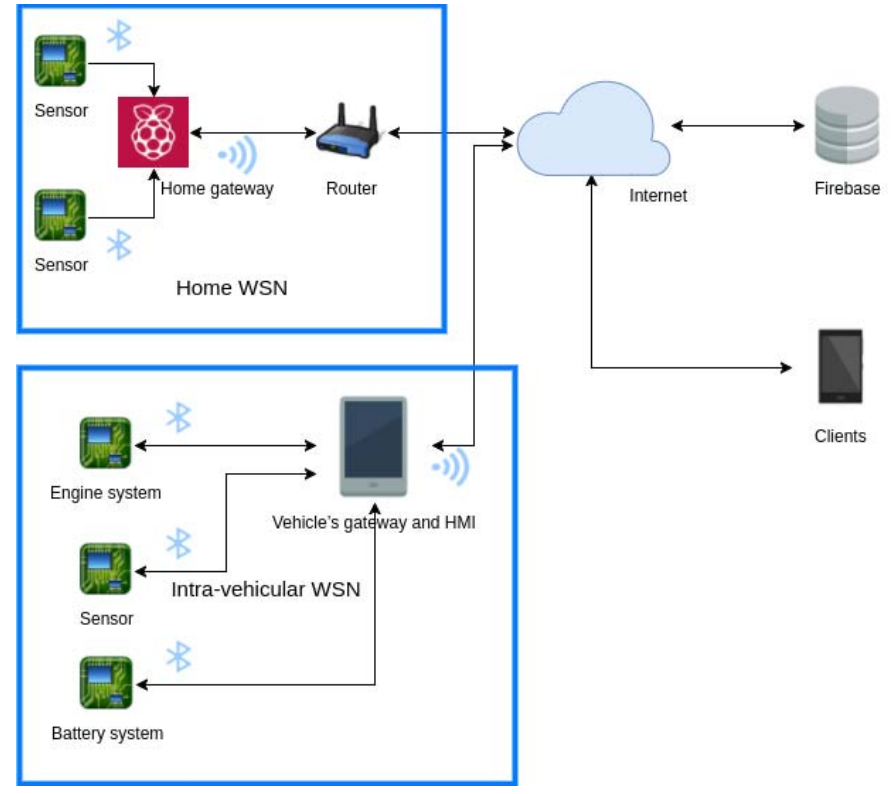

Fig. 2. Arquitecture of the proposed IoT system.

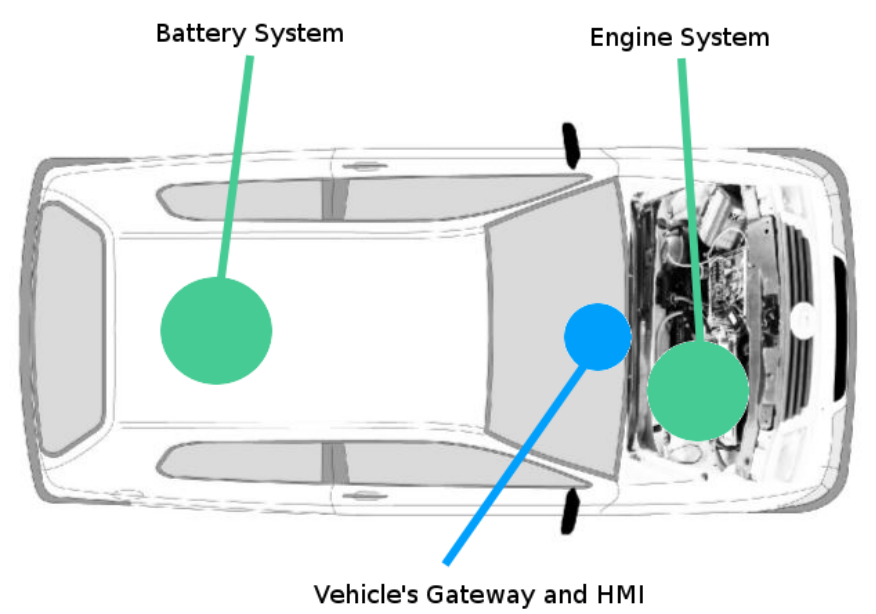

Fig. 3. Placement of the main IVWSN nodes inside of the car.

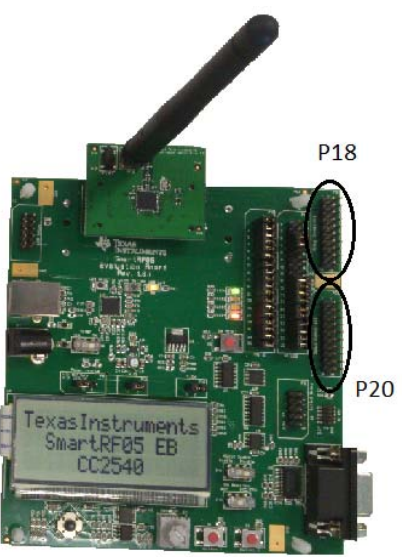

Fig. 4. BLE module used in the development of the IVWSN.

frames from the battery system have a similar structure. Data is transmitted every $500 \mathrm{~ms}$. 


\section{B. Vehicle's Gateway and Human-Machine Interface}

The vehicle's gateway and HMI may be either a smartphone or a tablet with mobile data connectivity. Besides the sensor nodes installed in the car, the internal sensors of this mobile device are also used to provide relevant information in the context of the proposed IoT system. For example, the GPS receiver is used to provide the vehicle's location and speed.

The first prototype was developed and tested using a smartphone model Motorola Moto G (2nd gen) with Android 6.0 and the Android Studio 2.1 IDE. The developed mobile app was created using mainly fragments, with the goal to simplify the reuse of the user interface components and to provide a flexible and organized user interface. It has four main activities: start menu, BLE device discovery, sensor data presentation and weather information. The periodic data reception is managed by a background service, allowing the user to perform other operations. The weather information is collected from the online service OpenWeatherMap. A screenshot of this mobile app is presented in the next section. The second prototype of the gateway will replace the smartphone by a tablet.

\section{Internet Server and Database}

In the proposed IoT system, sensor data collected from the vehicles is stored in a Firebase database using either a cellular network or a Wi-Fi network. In case there is no Internet connection available, data is cached locally. Once the connection comes up again, data is uploaded and the cache is cleared. This is done to save up storage in the mobile device. Using Firebase, we don't have to worry about data availability, so the cache is really just used as a backup plan.

As shown in Fig. 5, data is stored in JSON (JavaScript Object Notation) format. In this case, battery and the engine data are stored under a common key called "sensor". Firebase allows registering listeners for data change in real-time. In the client's mobile app, we subscribe to the sensor we want to listen to and we retrieve the changes in real-time.

Since the user will not be always using the client's app we can use the Firebase Cloud Messaging (FCM) to send a push notification when there is an important event, for example, a critical battery charge level. When this happens, the vehicle's tablet sends an HTTP request to a FCM endpoint that will trigger a notification in the clients that subscribed to that event.

Firebase's pricing model includes a free plan that is suitable for the development phase, since it provides several features, including FCM, real-time database and authentication providers for popular login methods (Google+, Facebook, email and password). The most important restriction for the production phase will be the limited number of concurrent connections to the real-time database, which are 100. However, the paid plans are not very expensive and there are plans on which users pay for the resources they use, which can turnout cheaper than initially thought. The use of Firebase is an alternative for the deployment of our own database servers using, for example, a MySQL relational database management system (RDBMS), which is a solution that we implemented in previous works [26].



Fig. 5. Example of the format of the sensor data stored in Firebase.

\section{Client Mobile App}

The client mobile app is currently under development using an Android smartphone. It will be able to connect to the Firebase database and have access to all sensor data uploaded by the vehicle's gateway in real-time. It will also allow the configuration of system parameters and the manual control of actuators in the vehicle, such as remotely turn on/off the battery charger.

\section{E. Residential Wireless Sensor Network}

The home WSN included in Fig. 2 is not an essential component of the proposed IoT system, but it allows some extra functionalities. In the current prototype, it is also based in BLE networks and uses a Raspberry Pi 3 model B as gateway. The BLE-enabled sensor nodes send their data to the gateway, which forwards the information to the Firebase database through the residence's Internet router.

The inclusion of the home WSN in the IoT architecture can allow, for example, the control the vehicle's battery charging dynamically, according to certain criteria, such as the cost of electricity (which can change according the hour of the day) or the current load in the residence, in order to avoid a current overload and the trigger of the main circuit breaker. In this case, a sensor of the home WSN may measure the home current consumption in real-time and control the vehicle's battery charging current dynamically through the Internet. This idea was already implemented in [25], but using a short-range point-to-point RF link, which constrains the distance between the home's current sensor and the battery charger. On the other hand, the use of the proposed IoT system eliminates all the distance restrictions.

\section{RESULTS AND DISCUSSION}

The first experimental tests presented in this section aim to evaluate the reliability of the BLE network in the transmission of data from the sensor nodes (peripheral stations) to the central station. All stations in these tests were CC2540EM modules running an embedded application that was developed based on the TI BLE stack version BLE-CC2540-1.3.2. After a BLE connection between the master (central station) and a slave (peripheral station) is established, time is divided into connection events [27]. For the notifications and indications 
modes, each connection event starts with a packet transmission from the master (poll), which is followed by a packet from the slave (response). After that, the connection event may terminate or otherwise continue with more alternate packets from the master and the slave, depending on the values of the configured parameters. When the slave latency parameter is equal to zero, the interval between active connection events is equal to the connection interval.

The first tests measured the packet error rate (PER) for the transmission of 1000 data packets using either notifications (which does not allow retransmissions) or indications (which support acknowledgements and retransmissions). The tests were performed using a transmission power of $0 \mathrm{dBm}$, the maximum allowed payload length ( 20 bytes $)$, which is the worst case, and a single data packet per BLE connection interval. The period of generation of data packets by the application, or packet period, was configured as a multiple of the connection interval, as explained below.

A set of tests was performed inside of the vehicle in order to assess the possibility of errors due to the effect of its metallic parts on the intensity of the signal or due to the interference from the electric motor on the transmitted signals. These tests were performed using notifications with both the connection interval and the packet period set to $500 \mathrm{~ms}$. The central station was placed at the car dashboard and the peripheral station placed at different locations on the vehicle. One test was made with the peripheral station placed close to the electric motor, with the hood closed, while the motor was accelerating. Similar tests were made with the peripheral station near the front lights, the rear lights, the battery system and the four tires. No packet errors were registered during these tests.

The following tests were performed with short distance $(1 \mathrm{~m})$ between the stations, without obstructions and without interference from Wi-Fi networks or other sources, in order to provide a controlled setup that excludes any other causes of packet errors except for limitations of the BLE stack.

With the notifications mode, it is possible to configure a packet period equal to the connection interval. Due to the characteristics of the indications mode, it is not advisable to do the same because for each data packet that the peripheral station sends it is necessary to wait for the reception of an ACK (acknowledgement) packet from the central station in the next connection event, which means that the packet period needs to be at least twice the value of the connection interval. This fact was confirmed in our tests, where the use of a packet period equal to the connection interval resulted in PER values around $54 \%$ regardless of the connection interval.

Even when the packet period was configured as twice of the connection interval, there were still errors due to limitations of the TI BLE stack implementation, with PER ranging from $25 \%$ to $3 \%$ for connection intervals ranging from $7.5 \mathrm{~ms}$ to $60 \mathrm{~ms}$, respectively. Only with connection intervals from $100 \mathrm{~ms}$ and above the packet errors were completed eliminated.

In the case that the master does not receive a data packet correctly, it does not send the ACK in the next connection event. Instead, the peripheral station (slave) retransmits the data packet at each subsequent connection event (after receiving the corresponding poll packet) until the ACK is



Fig. 6. Example of data packet retransmission with indications.

received. Fig. 6 shows an example of packet exchange between a master and a slave, which was captured using the TI SmartRF packet sniffer. Each slave data packet carries a 16-bit sequence number in its payload (which was used to enable the calculation of the PER in the tests). The connection interval was set to $100 \mathrm{~ms}$. The second transmission of the slave data packet (with sequence number "E5 00") corresponds to a retransmission, because the first transmission was not successful.

If the packet period is exceeded while waiting for the ACK, the peripheral station blocks the transmission of the next data packet, causing its loss. Therefore, in order to accommodate at least one retransmission with indications, it is necessary to make the packet period at least three times higher than the connection interval. Given these results, we conclude that for a reliable operation with the considered BLE platform using the indications mode, the minimum connection interval must be $100 \mathrm{~ms}$ and the minimum packet period must be $300 \mathrm{~ms}$.

The following tests concern the transmission of real data values from the sensor nodes to the vehicle's mobile device via BLE and the presentation of these values on the mobile device. Table I presents the sensors of the traction system and their main characteristics in terms of maximum expected value, unit and communication interface with the corresponding peripheral BLE module.

Fig. 7 presents an example of the traction system screen of the smartphone/tablet application that acts as the vehicle's gateway and HMI, showing the values of some of the sensors presented of Table I. The sensor ranges were configured with limits larger than the expected values specified on the table to accommodate a safety margin. The motor temperature sensor was not connected at the time of this test. A similar test was performed for the battery system, with analogous results.

\section{CONCLUSION}

This paper presented an IoT system that allows the users to monitor the data provided by the sensors available on a vehicle, and to control processes, such as the EV battery charging, either manually or automatically, anytime and anywhere.

Despite some performance limitations observed with the utilized BLE platform, the experimental results presented in this paper demonstrate that the wireless network is able to satisfy the requirements of the developed IVWSN, if its parameters are properly configured. Future work includes the development of new functionalities for the system. We also intend to evaluate new hardware and software BLE platforms and new BLE protocol versions for both residential and intravehicular WSNs. 
TABLE I. SENSOR CHARACTERISTICS FOR THE TRACTION SYSTEM.

\begin{tabular}{|c|c|c|c|}
\hline $\begin{array}{c}\text { Sensor } \\
\text { Data }\end{array}$ & $\begin{array}{c}\text { Maximum } \\
\text { Value }\end{array}$ & Unit & $\begin{array}{c}\text { Communication } \\
\text { Interface }\end{array}$ \\
\cline { 1 - 3 } $\begin{array}{c}\text { Controller } \\
\text { State }\end{array}$ & 3 & - & \multirow{2}{*}{} \\
\cline { 1 - 2 } $\begin{array}{c}\text { Controller } \\
\text { Current }\end{array}$ & 300 & $\mathrm{~A}$ & \multirow{2}{*}{ UART } \\
\cline { 1 - 2 } $\begin{array}{c}\text { Controller } \\
\text { Voltage }\end{array}$ & 300 & $\mathrm{~V}$ & \\
\cline { 1 - 2 } $\begin{array}{c}\text { Charge } \\
\text { State }\end{array}$ & 100 & $\%$ & \\
\cline { 1 - 2 } $\begin{array}{c}\text { Controller } \\
\text { Power }\end{array}$ & 50 & $\mathrm{~kW}$ & \\
\cline { 1 - 2 } $\begin{array}{c}\text { Controller } \\
\text { Temperature }\end{array}$ & 100 & ${ }^{\circ} \mathrm{C}$ & \\
\hline $\begin{array}{c}\text { Motor } \\
\text { Temperature }\end{array}$ & 100 & ${ }^{\circ} \mathrm{C}$ & \multirow{2}{*}{$\mathrm{ADC}$} \\
\hline
\end{tabular}

(1) CEPIUM - Engine

Controller voltage and current

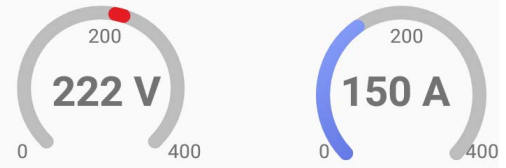

Controller power and temperature
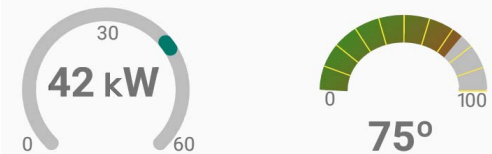

Change state



Motor temperature

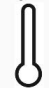

$0^{\circ}$

\section{$\triangleleft$}

\section{$\mathrm{O}$}

$\square$

Fig. 7. Vehicle's HMI screen for the traction system sensor data.

\section{ACKNOWLEDGEMENTS}

This work has been supported by COMPETE: POCI-01-0145FEDER-007043 and FCT - Fundação para a Ciência e Tecnologia within the Project Scope: UID/CEC/00319/2013.

\section{REFERENCES}

[1] D. Bandyopadhyay, J. Sen, "Internet of Things: Applications and Challenges in Technology and Standardization," SPRINGER Wireless Personal Communications, vol.58, no.1, pp.49-69, May 2011.

[2] Brahima Sanou. (2017, Mar. 10). "ICT Facts and Figures 2016" [Online]. Available: http://www.itu.int/en/ITU-D/Statistics/Documents /facts/ICTFactsFigures2016.pdf

[3] Dave Evans, "The Internet of Things - How the Next Evolution of the Internet Is Changing Everything," CISCO, pp.1-10, Apr. 2011.

[4] C. Rowland, E. Goodman, M. Charlier, A. Light, A. Lui, "Designing Connected Products: UX for the Consumer Internet of Things," O’Reilly, May 2015.

[5] António F. Maio, José A. Afonso, "Wireless Cycling Posture Monitoring Based on Smartphones and Bluetooth Low Energy," Lecture Notes in Engineering and Computer Science: Proceedings of the World Congress on Engineering, London, U.K., pp.653-657, July 2015.
[6] W. Z. Khan, Y. Xiang, M. Y. Aalsalem, Q. Arshad, "Mobile Phone Sensing Systems: A Survey," IEEE Communications Surveys \& Tutorials, vol.15, no.1, pp.402-427, First Quarter 2013.

[7] M. Di Natale, H. Zeng, P. Giusto, A. Ghosal, "Understanding and Using the Controller Area Network Communication Protocol: Theory and Practice," Springer Science \& Business Media, 2012.

[8] J. Lin, T. Talty, O. Tonguz, "On the Potential of Bluetooth Low Energy Technology for Vehicular Applications,” IEEE Commun. Mag., vol.53, no.1, pp.267-275, Jan. 2015.

[9] A. Pantelopoulos, N.G. Bourbakis, "A Survey on Wearable SensorBased Systems for Health Monitoring and Prognosis," IEEE Transactions on Systems, Man, and Cybernetics, Part C: Applications and Reviews, vol.40, no.1, pp.1-12, Jan. 2010.

[10] C. Buratti, A. Conti, D. Dardari, R. Verdone, "An Overview on Wireless Sensor Networks Technology and Evolution," MDPI Sensors, vol.9, no.9, pp.6869-6896, Aug. 2009.

[11] P. Bajpai. (2017, Mar. 11). "The Rise of Electric Vehicles: By The Numbers" [Online]. Available: http://www.nasdaq.com/article/the-riseof-electric-vehicles-by-the-numbers-cm595564

[12] S. Knupfer, R. Hensley, P. Hertzke, P. Schaufuss, N. Laverty, N. Kramer, "Electrifying Insights: How Automakers can Drive Electrified Vehicle Sales and Profitability," McKinsey\&Company, pp.1-28, Jan. 2017.

[13] D. Pedrosa, V. Monteiro, H. Gonçalves, J. S. Martins, J. L. Afonso, “A Case Study on the Conversion of an Internal Combustion Engine Vehicle into an Electric Vehicle," IEEE VPPC Vehicle Power and Propulsion Conference, pp.1-5, Oct. 2014.

[14] S. Kamath, J. Lindh, "Measuring Bluetooth Low Energy Power Consumption," Application Note AN092, Texas Instruments, pp.1-24, 2012.

[15] R. Want, B. N. Schilit, S. Jenson, "Enabling the Internet of Things," IEEE Computer, vol.48, no.1, pp.28-35, Jan. 2015.

[16] M. Siekkinen, M. Hiienkari, J. K. Nurminen, J. Nieminen, "How Low Energy is Bluetooth Low Energy? Comparative Measurements with ZigBee/802.15.4," IEEE WCNCW Wireless Communications and Networking Conference Workshops, pp.232-237, Apr. 2012.

[17] J. R. Lin, T. Talty, O. K. Tonguz, "An Empirical Performance Study of Intra-Vehicular Wireless Sensor Networks under WiFi and Bluetooth Interference," IEEE GLOBECOM Global Telecommunications Conference, pp.581-586, Dec. 2013.

[18] J. A. Afonso, A. J. F. Maio, R. Simoes, "Performance Evaluation of Bluetooth Low Energy for High Data Rate Body Area Networks," Wireless Personal Communications, vol.90, no.1, pp.121-141, Sept. 2016 .

[19] (2017, Mar. 10). "Android Auto" [Online]. Available: https://www.android.com/auto/

[20] J. Boman, J. Taylor, A. H. Ngu, "Flexible IoT Middleware for Integration of Things and Applications," IEEE CollaborateCom International Conference on Collaborative Computing: Networking, Applications and Worksharing, pp.481-488, Oct. 2014

[21] (2017, Mar. 10). "Global Sensor Networks - A Middleware for Processing Sensor Data in the Internet (since 2004)". [Online]. Available: http://lsir.epfl.ch/research/current/gsn

[22] Google, "Firebase | App success made simple" [Online]. Available: https://firebase.google.com

[23] A. Tahat, A. Said, F. Jaouni, W. Qadamani, "Android-Based Universal Vehicle Diagnostic and Tracking System," IEEE International Symposium on Consumer Electronics, pp.137-143, June 2012.

[24] Y. Leehter, Y. Q. Chen, W. H. Lim, "Internet of Things for Electric Vehicle: An Improved Decentralized Charging Scheme," IEEE International Conference on Data Science and Data Intensive Systems, pp.651-658, Dec. 2015.

[25] V. Monteiro, J. P. Carmo, J. G. Pinto, J. L. Afonso, "A Flexible Infrastructure for Dynamic Power Control of Electric Vehicle Battery Chargers," IEEE Transactions on Vehicular Technology, vol.65, no.6, pp.4535-4547, June 2016.

[26] Diogo S. Oliveira, José A. Afonso, "Mobile Sensing System for Georeferenced Performance Monitoring in Cycling", Lecture Notes in Engineering and Computer Science: Proceedings of the World Congress on Engineering, London, U.K., pp.269-273, July 2015.

[27] Bluetooth SIG, "Specification of the Bluetooth System. Master Table of Contents \& Compliance Requirements," Nov. 2003. 